\title{
Time to Integrate Malaria and Neglected Tropical Diseases Control and Elimination
}

\author{
Dirk Engels $^{1,2, *} ;$ Fang Huang ${ }^{2,3} ;$ Xiao-nong Zhou ${ }^{2,3,4}$
}

Traditionally, malaria has always been considered one of the tropical diseases. This only has changed in the late 1990s, with the World Health Organization (WHO) profiling HIV, tuberculosis (TB), and malaria as priority "poverty-related" killing diseases (1). This has led to the creation of the Global Fund to Fight AIDS, Tuberculosis, and Malaria (2), that has mobilized substantial resources to advance the control of all three diseases, even if mostly in a siloed manner.

Around the same time, efforts were undertaken to solicit international attention for an entire group of other tropical diseases, currently still affecting over 1.7 billion people worldwide, that had been left "neglected" (3). The global response that was mounted to advance the control/elimination of neglected tropical diseases (NTDs) - that are more disabling than killing diseases — was based on a different logic. It profiled NTDs as a group of diseases that 1) together represented a similar burden as $\mathrm{HIV}, \mathrm{TB}$, or malaria; 2) were treatable or preventable by similar interventions that could be delivered as integrated packages; and 3) were entrenched in poverty as no other diseases, urging the international community to prioritize the deployment of development efforts focused on the poorest sections of society.

Both global strategies have worked well, even though with vastly different levels of resources.

Impressive global progress has been made in the fight against malaria over the last two decades. The malaria case incidence rate (cases per 1,000 population at risk) has decreased from 80 in 2000 to 57 in 2019, a reduction of almost $30 \%$. The mortality incidence rate (deaths per 100,000 population at risk) was reduced from 25 in 2000 to 10 in 2019, or a reduction of $60 \%$. As a result, the global number of deaths due to malaria has fallen from 736,000 in 2000 to 409,000 in 2019, a decrease of over $40 \%$. Over the same period, the number of countries with fewer than 100 indigenous malaria cases increased from 6 to 27 , with 21 countries — including China - reporting zero indigenous malaria cases for at least three consecutive years, and 10 of these countries being certified malaria- free by the WHO (4). In 2019, an estimated 3.0 billion USD was spent globally on malaria control and elimination (5).

Interventions for NTDs, especially large-scale preventive treatments for lymphatic filariasis, onchocerciasis, schistosomiasis, soil-transmitted helminthiasis, and trachoma, have scaled up massively over the last decade with over a billion people reached yearly from 2015 to 2019 . This has led, at the end of 2020 , to already 600 million fewer people needing interventions for NTDs and 42 countries having eliminated at least one NTD. A complex disease such as sleeping sickness has been brought to the verge of elimination, elimination of visceral leishmaniasis is close to being achieved in South-East Asia, and the global number of reported cases of Chagas disease has decreased by $40 \%$ between 2011 and 2019. During the same period, 90 million people have been freed from the risk of vector transmission of Chagas disease, and universal blood screening for the disease implemented at $100 \%$ in Latin-America (6-7). The yearly amount of Overseaos Development Aid (ODA) funding has been estimated to be in the order of 300 million USD per year since 2014, leveraging a contribution in medicines donated by the pharmaceutical sector with a value of approximately 1.5 billion USD per year (8-9).

Despite good progress, both program areas face challenges. The global gains in combating malaria have levelled off in recent years. The emergence of resistance to insecticides and medicines is threatening malaria control and elimination (10), and the quest for novel technological solutions is hardly coping with the need. Redistributing resources toward control or eliminationspecific interventions, such as diagnosis and treatments, vector control, preventive treatments in pregnant women and children, and strengthening surveillance systems to identify and investigate foci, may produce economic efficiencies (5). Hence the increasing awareness that something more fundamentally preventive needs to be done, and new program and policy interventions to be developed, such as integrated vector management along with 
community involvement (11).

Likewise, notwithstanding the good progress in scaling up of NTD interventions, progress for NTDs such as leprosy and Guinea worm disease has been stagnating. Thanks to progress in $\mathrm{R} \& \mathrm{D}$ and implementation research interventions for NTDs such as Buruli ulcer, scabies, rabies, and yaws are now ready to be scaled up towards 2030, but considerable challenges remain in terms of affordable access to the required quantities of commodities and for technological solutions like novel diagnostics across the NTD spectrum. The need for novel tools and strategies is especially critical for five or six NTDs that remain relatively orphaned (12). These needs will not be fulfilled if the current siloed approach and funding logic in global health is maintained - hence the increasing awareness for the need to find synergies with other diseases and health areas in terms of implementation and $R \& D$ and the need for "development solutions" to limit the transmission of NTDs. The latter are particularly important for dengue and other Aedes-transmitted diseases, for which there are no other options than supportive treatment and vector control.

The above challenges provide an incentive for an integrated approach to interventions that are common to both disease areas, as illustrated by the recent convergence between the Zika virus outbreak and emerging evidence of pyrethroid resistance and loss of efficacy of long-lasting insecticidal nets (LLINs) in malaria control, that has prompted WHO to strongly advocate for integrated vector control in 2017 (13-14).

The recent coronavirus disease 2019 (COVID-19) pandemic has provided a further stark reminder that technological advancements are undeniably a big part of the solution (15), but not the only solution. It is equally important to reduce the risk or force of disease transmission, whether it be airborne, vector-borne, water-borne, food-borne, or zoonotic. This can only be achieved by acting simultaneously on human, animal, and environmental health through One Health approach, in an integrated manner.

An additional argument in favour of integration is surveillance. With an ever-increasing risk of emerging diseases, but also an increasing number of diseases that are on the verge of elimination and may resurge, it is important to put cost-effective surveillance methods in place. An integrated approach can greatly contribute to this, as illustrated by the example of sleeping sickness surveillance while doing rapid diagnosis of malaria in a primary healthcare setting (16).

The current state of advancement in technology has undoubtedly made integrated approaches more feasible. The recent epidemics of chikungunya, Ebola virus disease, and especially COVID-19 have shown that new technological solutions can rapidly be developed based on existing platforms, if will and resources are available.

Multi-disease solutions clearly have the potential to provide better return on investment. And opportunities for integration or convergence of malaria and NTD interventions exist, such as 1) environmental improvement aimed at control of transmission of multiple diseases $(17)$; 2) the setting up of integrated entomological and disease surveillance and response systems; 3) potential integration of WHOrecommended preventive therapies for malaria* with preventive treatment activities against NTDs, and potentially with other programs such as HIV, female genital schistosomiasis and cervical cancer prevention and treatment programs (18); and 4) the development of new or improved tools with dual- or multi-disease utility such as new formulations of ivermectin targeting residual outdoor transmission of malaria, onchocerciasis, lymphatic filariasis and scabies, or multiplex diagnostics, both for differential diagnosis (of fevers for example) or surveillance

In conclusion, the COVID-19 pandemic has made the world realize that a more systemic approach to solutions is required (19), if we wish to "build back better" in a sustainable way (20). In this endeavour, it would be wrong to focus on epidemics only, let alone on airborne epidemics only. Inequity in health across the disease spectrum needs to be considered. Furthermore, along with battered global economies, there is likely to be less ODA funding available in the foreseeable future. It will be important to try and go further with less being available. Integrated approaches may help to achieve this, and a more integrated approach to the control and elimination of malaria and NTDs may provide an iconic example of how this could be done.

doi: $10.46234 / \mathrm{ccdcw} 2021.100$

\# Corresponding author: Dirk Engels, engelsd54@gmail.com.

${ }^{1}$ Secretariat, Uniting Forum To Combate NTDs, Geneva, Switzerland;

\footnotetext{
* such as intermittent preventive treatment of pregnant women (IPTp), intermittent preventive treatment of infants (IPTi), and seasonal malaria chemoprevention (SMC).
} 
${ }^{2}$ NHC Key Laboratory for Parasite and Vector Biology, National Institute of Parasitic Diseases, Chinese Center for Disease Control and Prevention, Shanghai, China; ${ }^{3}$ Chinese Center for Tropical Diseases Research; WHO Collaborating Centre for Tropical Diseases; ${ }^{4}$ School of Global Health, Chinese Center for Tropical Diseases Research, Shanghai Jiao Tong University School of Medicine, Shanghai, China.

Submitted: April 19, 2021; Accepted: April 22, 2021

\section{REFERENCES}

1. World Health Organization. Global report for research on infectious diseases of poverty. 2012. https://www.who.int/tdr/capacity/global_ report/en/. [2021-04-01].

2. Kerouedan D. Enjeux politiques de l'evaluation du Fonds mondial de lutte contre le sida, la tuberculose et le paludisme. Med Trop 2010; $70: 19-27$.

3. Engels D, Zhou XN. Neglected tropical diseases: an effective global response to local poverty-related disease priorities. Infect Dis Poverty 2020;9(1):10. http://dx.doi.org/10.1186/s40249-020-0630-9.

4. World Health Organization. World malaria report 2020. https://www. who.int/teams/global-malaria-programme/reports/world-malaria-report2020. [2021-04-01].

5. Shretta R, Liu J, Cotter C, et al. Malaria elimination and eradication. In: Holmes KK, Bertozzi S, Bloom BR, et al., editors. Major Infectious Diseases. 3rd ed. Washington, DC: The International Bank for Reconstruction and Development/The World Bank. 2017.

6. World Health Organization. Neglected Tropical Diseases progress dashboard 2011-2020. Geneva: WHO. https://www.who.int/teams/ control-of-neglected-tropical-diseases/overview/progress-dashboard2011-2020. [2021-04-01].

7. Uniting to Combat Neglected Tropical Diseases. Progress on the London Declaration on NTDs. West Sussex: Uniting to Combat NTDs. https://unitingtocombatntds.org/progress/. [2021-04-01].

8. World Health Organization. Investing to overcome the global impact of neglected tropical diseases: Third WHO report on neglected tropical diseases. Geneva: WHO. http://apps.who.int/iris/bitstream/handle/ 10665/152781/9789241564861_eng.pdf;jsessionid=61384725EE69B7 3645557869AE9FDF2A?sequence=1. [2021-04-1].

9. Cohen JP, Silva L, Cohen A, Awatin J, Sturgeon R. Progress report on neglected tropical disease drug donation programs. Clin Ther 2016;38(5):1193 - 204. http://dx.doi.org/10.1016/j.clinthera.2016.02. 031 .

10. The malERA Refresh Consultative Panel on Insecticide and Drug Resistance. malERA: an updated research agenda for insecticide and drug resistance in malaria elimination and eradication. PLoS Med 2017;14(11):e1002450. http://dx.doi.org/10.1371/journal.pmed.1002 450.

11. Hemingway J. The role of vector control in stopping the transmission of malaria: threats and opportunities. Philos Trans R Soc Lond B Biol Sci 2014;369(1645):20130431. http://dx.doi.org/10.1098/rstb.2013. 0431.

12. World Health Organization. Ending the neglect to attain the sustainable development goals: a road map for neglected tropical diseases 2021-2030. 2020. https://www.who.int/publications/i/item/ 9789240010352. [2021-04-01].

13. Alonso P, Engels D, Reeder J. Renewed push to strengthen vector control globally. Lancet 2017;389(10086):2270 - 1. http://dx.doi.org/ $10.1016 /$ S0140-6736(17)31376-4.

14. World Health Organization. Global vector control response 2017-2030. 2017. https://www.who.int/vector-control/publications/ global-control-response/en/. [2021-04-01].

15. Renu N. Technological advancement in the era of COVID-19. SAGE Open Med 2021;9:20503121211000912. http://dx.doi.org/10.1177/ 20503121211000912.

16. Lumbala C, Matovu E, Sendagire H, Kazibwe AJN, Likwela JL, Mavoko HM, et al. Performance evaluation of a prototype rapid diagnostic test for combined detection of gambiense human African trypanosomiasis and malaria. PLoS Negl Trop Dis 2020;14(4): e0008168. http://dx.doi.org/10.1371/journal.pntd.0008168.

17. Engels D. Fighting NTDs in burundi. 2021. https://rstmh.org/newsblog/blog/fighting-ntds-in-burundi. [2021-04-01].

18. Engels D, Hotez PJ, Ducker C, Gyapong M, Bustinduy AL, Secor WE, et al. Integration of prevention and control measures for female genital schistosomiasis, HIV and cervical cancer. Bull World Health Organ 2020;98(9):615 - 24. http://dx.doi.org/10.2471/BLT.20.252270.

19. Horton R. Offline: COVID-19 is not a pandemic. Lancet 2020;396 (10255):874. http://dx.doi.org/10.1016/S0140-6736(20)32000-6.

20. Ehrenberg N, Ehrenberg JP, Fontes G, Gyapong M, Rocha EMM, Steinmann $\mathrm{P}$, et al. Neglected tropical diseases as a barometer for progress in health systems in times of COVID-19. BMJ Glob Health 2021;6(4):e004709. http://dx.doi.org/10.1136/bmjgh-2020-004709. 